\title{
A QEE-Oriented Fair Power Allocation for Two-tier Heterogeneous Networks
}

\author{
Shiyu $\mathrm{Ji}^{1}$, Liangrui Tang ${ }^{1}$ Yanhua $\mathrm{He}^{1}$, Shuxian $\mathrm{Li}^{1}$ and Shimo $\mathrm{Du}^{2}$ \\ ${ }^{1}$ State Key Laboratory of Alternate Electrical Power System, North China Electric Power University \\ Beijing, 102206 - China \\ Fie-mail:15210726562@163.com] \\ ${ }^{2}$ China mobile communication corporation $\mathrm{H}$ angzhou branch \\ Hangzhou, 310006- China \\ [e-mail: dushimo@163.com] \\ *Corresponding author: Shiyu Ji
}

Received July 11, 2017; revised September 17, 2017; accepted October 8, 2017;

published May 31, 2018

\begin{abstract}
In future wireless network, user experience and energy efficiency will play more and more important roles in the communication systems compared to their roles at present. Quality of experience (QoE) and Energy Efficiency (EE) become the widely used metrics. In this paper, we study a combinatorial problem of QoE and EE and investigate a fair power allocation in heterogeneous networks. We first design a new metric, QoE-aware EE (QEE) to reflect the relationship of QoE and energy. Then, the concept of Utopia QEE is introduced, which is defined as the achievable maximum QEE in ideal conditions, for each user. Finally, we transform the power allocation process to an optimization of ratio of QEE and Utopia QEE and use invasive weed optimization (IWO) algorithm to solve the optimization problem. Numerical simulation results indicate that the proposed algorithm can get converged and efficiently improve the system energy efficiency and the QoE for each user.
\end{abstract}

Keywords: QEE, power allocation, two-tier heterogeneous networks, IWO

This research was supported by the Beijing Natural Science Foundation (4142049), National High Technology Research and Development of China 863 Program (2014AA01A701) and the Fundamental Research Funds for the Central Universities of China (2015XS07). 


\section{Introduction}

$\mathbf{N}_{\text {etwork isomerization is becoming one of the architectural shifts towards the emerging }}$ 5G wireless networks to support the ever-increasing need of data and services from users $[1,2]$. The paradigm shift towards heterogeneity brings many new challenges to wireless network design [3,4]. In future wireless network, a wide range of services can be offered to mobile users and the capacity of today's network is far away from satisfaction, which will finally affect the QoE for users. Thus, one of the main challenges is to promote the QoE of users with limited resource [5,6]. On the other hand, from the operator's perspective, macro-cells and small cells would be ultra-dense because of the huge traffic load in future, which will cause lots of problems (e.g. high energy cost). Considering these financial and environmental problems, energy efficiency is also necessary to be paid attention to $[7,8]$. In this paper, how to properly realize power allocation to improve the energy efficiency (EE) and QoE under such environment is our primary goal.

\section{A Related Work}

Nowadays, a substantial amount of work focuses on the areas of power allocation. The main purpose of these literatures is to improve the spectrum or energy utilization $[9,10]$. To improve the spectrum utilization, the literatures address the optimality of system capacity [11-17]. The authors in [11] propose a power allocation scheme to maximize the sum-rate of network. The scheme formulates a standard non-convex quadratically constrained quadratic problem (QCQP) and uses a distributed algorithm to solve it. In [12], the authors also propose an optimal power allocation to maximize the capacity of network. It assumes that the single cell power is constrained and formulates the optimal problem to get the optimal power allocation scheme. However, the interference among small cells is ignored in a dense environment $[13,14]$. The authors in $[13]$ use game theory to promote the transmission rate of edge users. However, these literatures fail to pay attention to the energy efficiency problem in a wireless environment.

Nowadays, with the densification of the network, there have been extensive researches on energy efficiency (EE)-oriented problems [18-22]. The system energy efficiency is studied in [18], in which a resource allocation scheme is proposed where base stations jointly process the data of all users to maximize the system EE. In [19], the authors not only consider the energy efficiency, but also consider to maintain the quality of service (QoS) level and formulate the power allocation scheme as a tractable convex optimization problem, which is solved by iterative algorithm. Non-cooperative game-theoretic approaches are also employed in [20] and [21] to handle the energy efficient power allocation problems in wireless networks.

As one of the common points, research works (and the references therein) only care for the EE of system and do not consider the EE of the individual users. This is called network EE-optimal problems (NEPs). The system would bring benefit to good channel condition 
users, and thus, the improvement in network EE is gained at the cost of benefit of users in the bad channel conditions. As result, the NEPs ignore the fairness of users in terms of EE. Therefore, the authors in [23] explore a max-min EE-optimal problem to ensure the EE of the worst-case user and propose a general EE-based update algorithm to tackle the max-min EE-optimal problem. However, the users' quality of experience is not considered in aforementioned work.

In future wireless system, users will not only consider the EE but also pursue the QoE. Actually, there have been some attempts on QoE-oriented. The authors propose lots of QoE models of users with different services [24]. In [25], the authors also investigate the optimal the sum of QoE and the proportional fair of users' QoE in [26]. However, these works all ignore to take consideration of algorithm design related to the EE and QoE. Since, different users will have different QoE and EE requirements, the QoE and EE for each user should be individually considered, rather than as a whole. So, in this paper, we investigate the power allocation in heterogeneous network to improve the QoE and EE of each user. We also design a metric called QoE-aware energy efficiency to measure the QoE and EE of each user.

\section{B Contribution}

In this work, our aim is to improve the EE and QoE of each user in a fair manner by power allocation. The main contribution of our work is summarized as follows.

- We investigate the user experience and formulate the different QoE functions for various type of users. The QoE function reflects the user satisfaction to the network.

- A new model called QEE is formulated in this paper, which is used to measure the QoE and EE of each user.

- We propose a metric to measure the user's proximity degree to the ideal value i.e., utopia QEE and formulate an optimal problem using it to avoid discriminating to bad users in power allocation.

- The IWO algorithm is proposed to solve the problem, and the performance of our proposed algorithm is verified by simulations.

The rest of this paper is organized as follows. In section 2, we introduce the system model and design the QoE and utopia QEE of each user with different services. In section 3, the problem is formulated and solved by IWO. Section 4 presents simulation experiments and analyzes the performances of the proposed algorithm. Finally, we draw the conclusion in Section 5.

\section{System model and QoE}

In this section, we firstly describe the model of two-tier heterogeneous networks, and then the QoE is proposed. 


\section{A System and Channel model}

As shown in Fig. 1, we consider two-tier heterogeneous networks with a macro-cell and $M$ small-cells. The set of cells can be denoted by $\mathcal{M}=\{1,2, \ldots, \boldsymbol{M}+\mathbf{1}\}$. We also define $\mathcal{N}=\{1,2, \ldots, N\}$ and $\mathcal{K}=\{1,2, \ldots, \mathrm{K}\}$ as the set of users and sub-channel, respectively. All users randomly distribute in the two-tier heterogeneous network. A sub-channel in each cell would be allocated to a user for transmission. We assume that; (i) $N=K(M+1)$, i.e., there are $K$ users per cell and sub-chann el; (ii) the $n$th user accesses to the $m$ th cell in $k$ th sub-channel for both macro-cell and small-cell. These assumptions are solely for the sake of notational simplicity. Our work can be extended to the case of arbitrary values $M, N, K$. The bandwidth of the sub-channel is $B$ and the transmission power of the sub-channel in $m$ th cell in $k$ th sub-channel is $p_{m}^{k}$.We also assume that the users would use different applications which transmit different services. In this paper, assuming that there is a central controller in two-tier heterogeneous networks, the controller can allocate the power for all users. The central controller has all the channel side information (CSI) and achieves good performance.

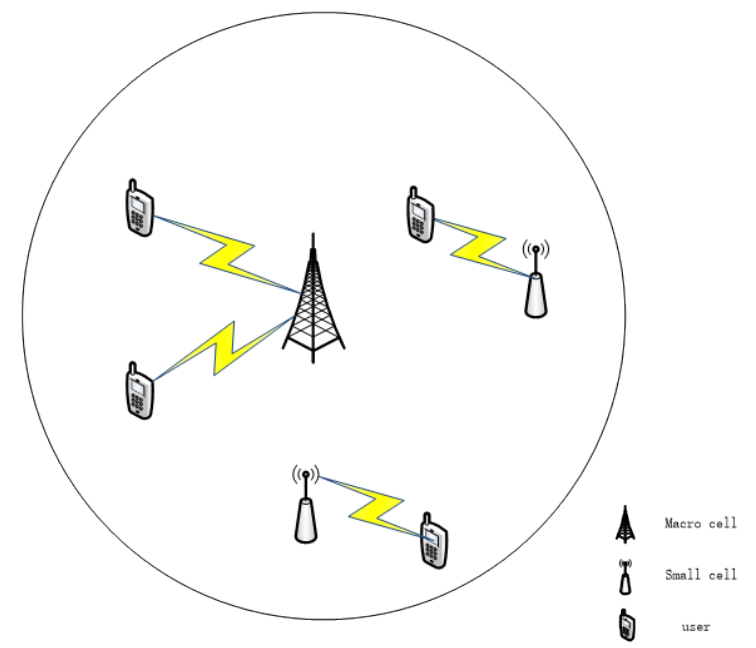

Fig. 1. System model

\section{B QoE with Different service}

In wireless networks, QoE assessment is a challenge which draws a lot of interests from both academia and industry. The Mean Opinion Score (MOS) model is widely used to characterize QoE whose value can reflect users' opinions on services, which ranges from totally unacceptable to fully satisfied [5]. We denote the QoE with C type and S type service.

Traditionally, the main C type service is voice service which usually measured by Perceptual Evaluation of Speech Quality (PESQ) recommended by International Telecommunication Union (ITU). However, it is still not suitable for dynamic real-time 
system because of the computationally complexity and requirement of the original speech signal. In [27], the QoE model for voice service is described as the function of transmission rate. We assume that the transmission rate of $\mathrm{C}$ type service is $R_{C}$, and its QoE will increase with the increasing transmission rate but the increasing rate will slow down. Thus, the function of the service can be denoted as:

$$
M O S= \begin{cases}0 & , R<R_{\min }^{r e q} \\ a \log (R / b) & , R_{\min }^{r e q} \leq R<R_{\max }^{r e q} \\ 5 & , R \geq R_{\min }^{r e q}\end{cases}
$$

where, $R_{\min }^{r e q}$ and $R_{\max }^{r e q}$ are the minimum and the maximum requirement transmission rates of $C$ type service, respectively. $a$ and $b$ are the constant value.

The $\mathrm{S}$ type services include real-time and non-real-time ones, such as video service which is a real-time service with adaptive coding and file download service which is a nonreal-time service. As described in [28], these services can use S-shaped function to reflect the satisfaction degree of users. The QoE value will not decrease to zero directly when the transmission rate is smaller than the required rate. We assume that the transmission rate of S type service is $R_{S}$. The obtained QoE of it can increase with the increasing of transmission rate and the increase speed gets faster firstly and slow down lately. Thus the function of the $\mathrm{S}$ type service is:

$$
M O S=\left\{\begin{array}{cl}
p^{2} a \log \left(R_{\mathrm{tan} \_1} / b\right) & \text { if } R<R_{\mathrm{tan} \_1} \\
\text { where } R=2 p(1-p) R_{\mathrm{int} \_1}+p^{2} R_{\mathrm{tan} \_1} & \text { if } R_{\mathrm{tan} \_1} \leq R<R_{\mathrm{tan} \_2} \\
a \log (R / b) & \text {,if } R_{\mathrm{tan} \_2} \leq R<R_{\mathrm{max}}^{\text {req }} \\
(1-p)^{2} a \log \left(R_{\mathrm{tan} \_2} / b\right)+10 p(1-p)+5 p^{2} & \text {, otherwith } \\
\text { where } R=(1-p)^{2} R_{\mathrm{tan} \_2}+2 p(1-p) R_{\mathrm{int} \_2}+p^{2} R_{\mathrm{max}} & \\
5 &
\end{array}\right.
$$

where, $R_{\text {tan_1 }}$ and $R_{\text {tan_2 }}$ are the points of contact in the curve MOS $=a \log (R / b) . p$ is a variate value.

\section{The Proposed Power Allocation Algorithm}

In this section, we develop a power allocation algorithm to achieve the optimal QoE-aware energy efficiency in a fair allocation manner.

\section{A Utopia QEE}

Before formulating the problem, we first discuss the Utopia QEE and the method to calculate the Utopia QEE for each individual user. 
Definition 1 (Utopia QEE): The Utopia QEE for each user can be defined as the maximal QEE it can be achieved in ideal conditions (without interference).

Accordingly, the following optimization problem can be formulated for $n$th user to obtain the Utopia QEE of $n$th user.

$$
\begin{aligned}
& \max \eta_{n}=\operatorname{MOS}_{n}^{I N} /\left(\varepsilon_{m} p_{m}^{k}+p_{n}^{f i x}+p_{m, k}^{I}\right) \\
& \text { subject to } \\
& \qquad C_{1}: p_{m}^{k} \leq p_{m, \max } \\
& C_{2}: p_{m}^{k}>0
\end{aligned}
$$

where, $\eta_{n}$ is Utopia QEE of $n$th user, $p_{n}^{f i x}$ is the fix power of $n$th user when accessing to the $m$ th cell, $p_{m}^{I}$ is the fixed power consumption of $k$ th sub-channel in $m$ th cell. $\varepsilon_{m}$ denotes the inverse of power amplifier efficiency of $m$ th cell. In ideal conditions, the $n$th user can make full use of power resource of $m$ th cell, so the constraint can be formulated as $C_{1} \cdot M_{n} S_{n}^{I N}$ is the QoE of $n$th user in ideal conditions which is only related to the transmission rate according to the formulation (1) and (2). The transmission rate in ideal conditions when the $n$th user accesses to the $m$ th cell can be calculated by:

$$
R_{n}^{I N}=B \log \left(1+p_{m}^{k} g_{m, n}^{k} /\left(N_{0} B\right)\right)
$$

where, $R_{n}^{I N}$ is the transmission rate in ideal conditions , $g_{m, n}^{k}$ is the channel power gain between $n$th user and $m$ th cell in $k$ th sub-channel. $N_{0}$ is the power spectral density (PSD) of the additive white Gaussian noise (AWGN). To simplify the formulation (3), we assume that $\mathcal{P}_{n}$ is the power consumption of the $n$th user when access to $m$ th cell in $k$ th sub-channel, which can be calculated by

$$
\mathcal{P}_{n}=\varepsilon_{m} p_{m}^{k}+p_{n}^{f i x}+p_{m, k}^{I}
$$

Thus, the optimization problem above can be transformed and the objective function can be rewritten as:

$$
\begin{aligned}
& \max \eta_{n}=\operatorname{MOS}_{n}^{I N} / \mathcal{P}_{n} \\
& \text { subject to } \\
& \qquad \begin{array}{c}
C_{1}: p_{m} \leq p_{m, \max } \\
C_{2}: p_{m}>0
\end{array}
\end{aligned}
$$

The optimization problem above is a convex-concave fractional programming. To find its global optimum, we define

$$
q\left(\eta_{n}\right)=\operatorname{MOS}_{n}^{I N}-\eta_{n} \mathcal{P}_{n}
$$

Then we have the following theorem, which can be proved by directly following a similar approach as that in [29].

Theorem 1: The Utopia QEE is achieved if 


$$
\max _{R_{n}^{I N}} q\left(\eta_{n}^{0}\right)=0
$$

Note that $q\left(\eta_{n}\right)$ only has a single variable, therefore, its maximum can be easily achieved by derivation methods, the detailed procedures to achieve the Utopia QEE are shown in the Table 1.

Table 1. The algorithm to achieve the Utopia QEE for $n$th user

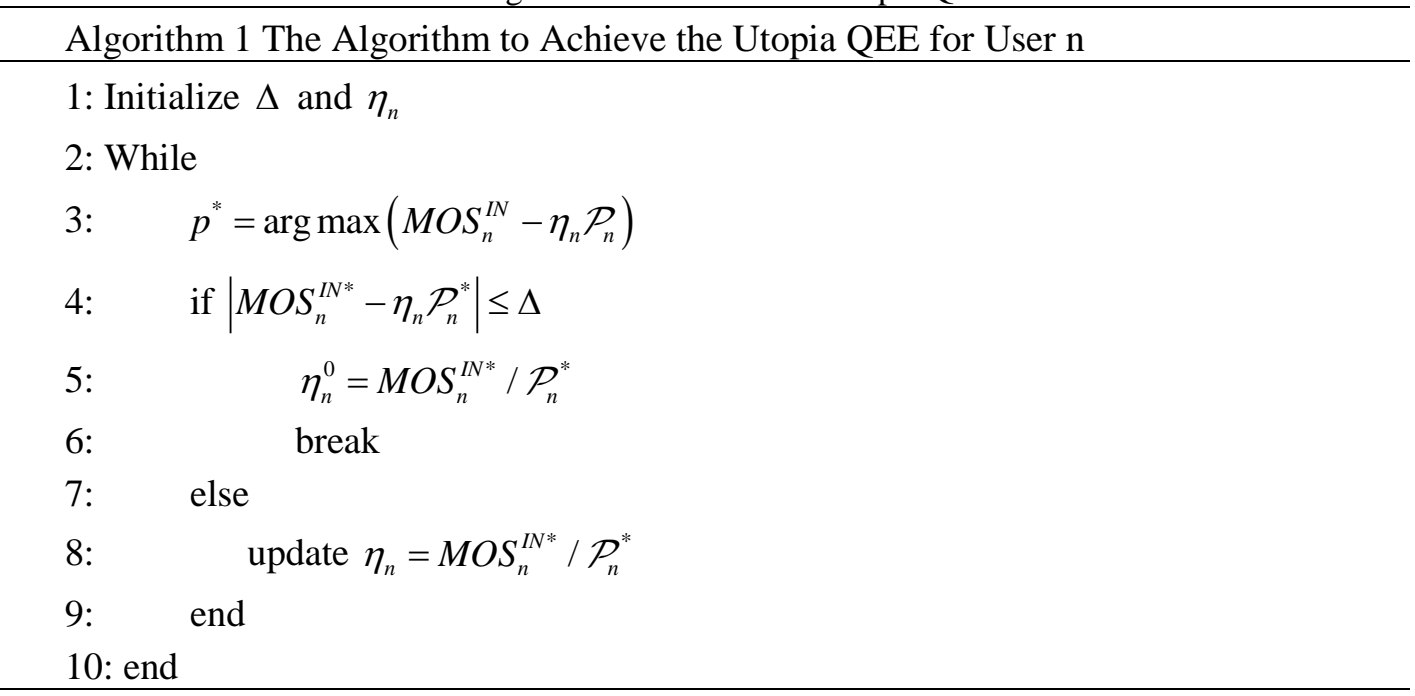

\section{B Problem Formulation}

In section 2, the Utopia QEE of each user has been achieved. In this section, we formulate our optimal problem. Our goal is to assign users to cells in a fair manner; i.e., the assignment allocates each user sufficient power without unduly restricting the amount of power available to others. To achieve the goal, we define a proximity degree for each user.

Definition 2 (proximity degree): The proximity degree for each user can be defined as the ratio of the actual QEE and the Utopia QEE.

The proximity degree is the metric to measure the benefit of $n$th user. When the proximity degree is higher, the user will get closer to the ideal condition. Our aim is to get the maximum proximity degree of all users which can also maximize the total QEE in a fair manner. The optimal problem can be formulated as: 


$$
\begin{aligned}
& \max \sum_{n=1}^{N}\left(Q E E_{n} / \eta_{n}\right) \\
& \text { subject to } \\
& \qquad C_{1}: \sum_{k \in \mathcal{K}} p_{m}^{k}<p_{m \max }, \forall m \in \mathcal{M} \\
& C_{2}: \frac{h_{m n} p_{m}^{k}}{\sum_{j \in \mathcal{N}, j \neq n} h_{j n} p_{j}^{k}+N_{0} B} \geq \gamma_{n \min }, \forall n \in \mathcal{N} \\
& C_{3}: p_{m}^{k}>0
\end{aligned}
$$

In formulation (9), $C_{1}$ and $C_{3}$ are the power constraints: The power cannot exceed the maximum power $p_{m \text { max }}$. $p_{m \text { max }}$ not only controls the out-cell interference, but also restricts the power amplifier to linear region which corresponds to the constant amplifier efficiency. $C_{2}$ is the QoS constraint: The SINR at received terminals should meet the requirement to maintain its performance. $\gamma_{n \min }$ is the recognition SINR of receiving set. $Q E E_{n}$ can be calculated by :

$$
Q E E_{n}=\operatorname{MOS}_{n} /\left(\varepsilon_{m} p_{m}^{k}+p_{n}^{f i x}+p_{m, k}^{I}\right)
$$

where $\mathrm{MOS}_{n}$ is the QoE of $n$th user which is related to the transmission rate with interference $R_{n}$. It can be described by:

$$
R_{n}=B \log \left(1+\frac{h_{m n} p_{m}^{k}}{\sum_{j \in \mathcal{M}, j \neq m} h_{j n} p_{j}^{k}+N_{0} B}\right)
$$

where, $h_{m n}$ and $h_{j n}$ are the channel gain between $m$ th and $j$ th base-station to $n$th user, respectively. For macro-cell users, the interferences are between small-cells and macro-cell users, and for small-cell users, the interferences have two parts, one is between other small-cells and small-cell user and the other is macro-cell and small-cell user.

\section{The Proposed Algorithm}

Above all, our target is to maximize the total QEE in a fair manner, thus, the problem is formulated as formulation (9), considering that the feasible solution space of this optimization problem is huge, it is difficult to find the optimal solution using enumeration method. So the practical algorithm should have fast convergence speed to get the optimal solution. In this paper, we use the Invasive Weed Optimization (IWO) to solve the optimal problem. The IWO has strong robustness to achieve the optimal value. The key methods can be summarized as follows: 


\section{Initialization}

Set the generation counter $t=1$ and generate the initial location of invasive weed which represents the transmission power in th iteration. The coordinate of location is the transmission power. The location can be denoted by

$$
P(t)=\left\{p_{1}^{1}(t), \ldots, p_{1}^{K}(t), \ldots p_{m}^{k}(t), \ldots, p_{M}^{K}(t)\right\}
$$

\section{Reproduction}

According to the object value in formulation (9), the number of seeds has a positive relationship with the object value. The seed number of each invasive weed can be calculated by:

$$
w=\left(f-f_{\min }\right)\left(s_{\max }-s_{\min }\right) /\left(f_{\max }-f_{\min }\right)+s_{\min }
$$

where, $f_{\max }$ and $f_{\min }$ are the maximum and minimum object value, respectively, $s_{\max }$ and $s_{\min }$ are the maximum and minimum seed number of one invasive weed can generate, respectively.

\section{Spatial Diffusion}

The seed will grow into a weed which will distrust around the father invasive weed according to the Normal distribution with zero mean. The standard deviation should decrease with the increase of iteration. The standard deviation value can be generated by:

$$
\sigma_{t}=\left(t_{\max }-t\right)^{x}\left(\sigma_{\text {inital }}-\sigma_{\text {final }}\right) / t_{\max }+\sigma_{1}
$$

where, $t_{\max }$ is the maximum iteration, $\sigma_{\text {inital }}$ and $\sigma_{\text {final }}$ are the initial and final standard deviation value, respectively. $x$ is the nonlinear harmonic factor.

\section{Selection}

When the weed number get equal to the maximum number $N_{u m} m_{\max }$, we select the $\mathrm{Num}_{\text {max }}$ weed with roulette method. The detail description is displayed in Fig. 2. 


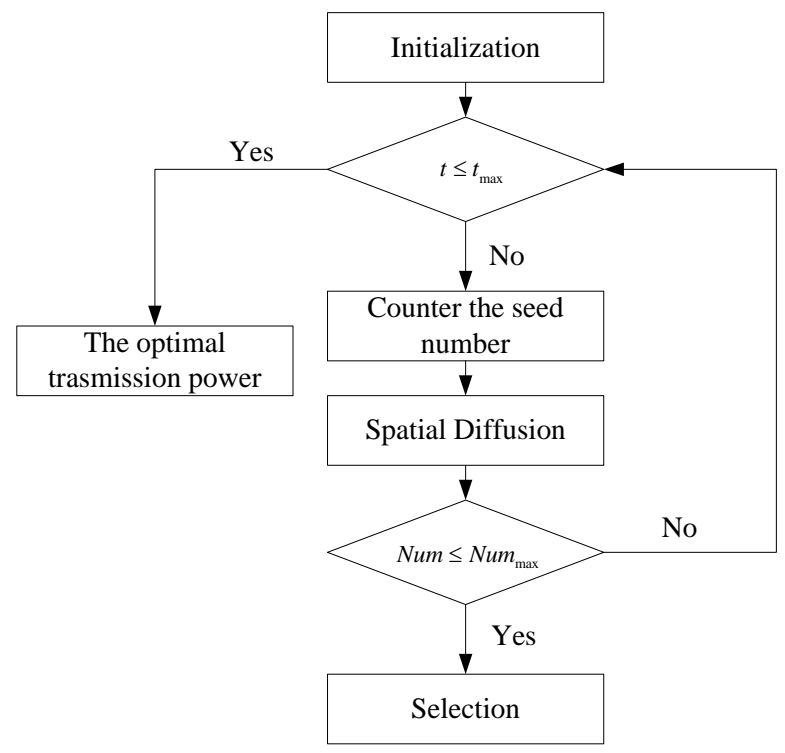

Fig. 2. The algorithm for maximum total proximity degree

\section{Simulation Experiment and Result Analysis}

The previous section is based on an analysis of the optimization problem in theory. In this section, the accuracy of the analysis is evaluated experimentally. To solve the optimization problem, we choose the invasive weed optimization algorithm. Here, the performance of the proposed algorithm is compared with that of other algorithms.

All the experiments are performed in the same simulation scenario shown in Fig. 1. The coverage radius of the macro-cell is $500 \mathrm{~m}$, and LPN is $10 \mathrm{~m}$. Each user is randomly distributed in the network. The experiment parameters are showed in Table 2 [30-32]

Table 2. Simulation parameters

\begin{tabular}{ll}
\hline Parameter & value \\
\hline Sub-channel Bandwidth(B) & $200 \mathrm{KHz}$ \\
Number of sub-channels(K) & 10 \\
the PSD of AWGN $\left(N_{0}\right)$ & $-174 \mathrm{dBm} / \mathrm{Hz}$ \\
Maximum transmit power of macro cell $p_{1 \max }$ & $10 \mathrm{w}$ \\
Maximum transmit power of small cell $p_{\text {mmax }}$ & $1 \mathrm{w}$ \\
the inverse of power amplifier efficiency $\varepsilon_{m}$ & 1 \\
The covering radio of macro-cell & $500 \mathrm{~m}$ \\
The covering radio of small cell & $10 \mathrm{~m}$ \\
The fixed power of macro-cell $p_{1, k}^{I}$ & $0.2 \mathrm{w}$
\end{tabular}


The fixed power of small cell $p_{m, k}^{I}$ $0.05 \mathrm{w}$

The fixed power of user $p_{n}^{\text {fix }}$ $30 \mathrm{mw}$

Maximum seed number of one invasive weed $s_{\max }$ 5

Minimum seed number of one invasive weed $s_{\max }$ 0

Initial standard deviation value $\sigma_{\text {inital }}$

Final standard deviation value $\sigma_{\text {final }}$

0.05

Maximum number of weed 100

\section{A Performance of IWO}

Fig. 3 shows the variation of object value with the iteration increasing, it is obvious that when the iteration comes to 5, the IWO falls into local optimization trap and gets converged when the iteration is around 15.

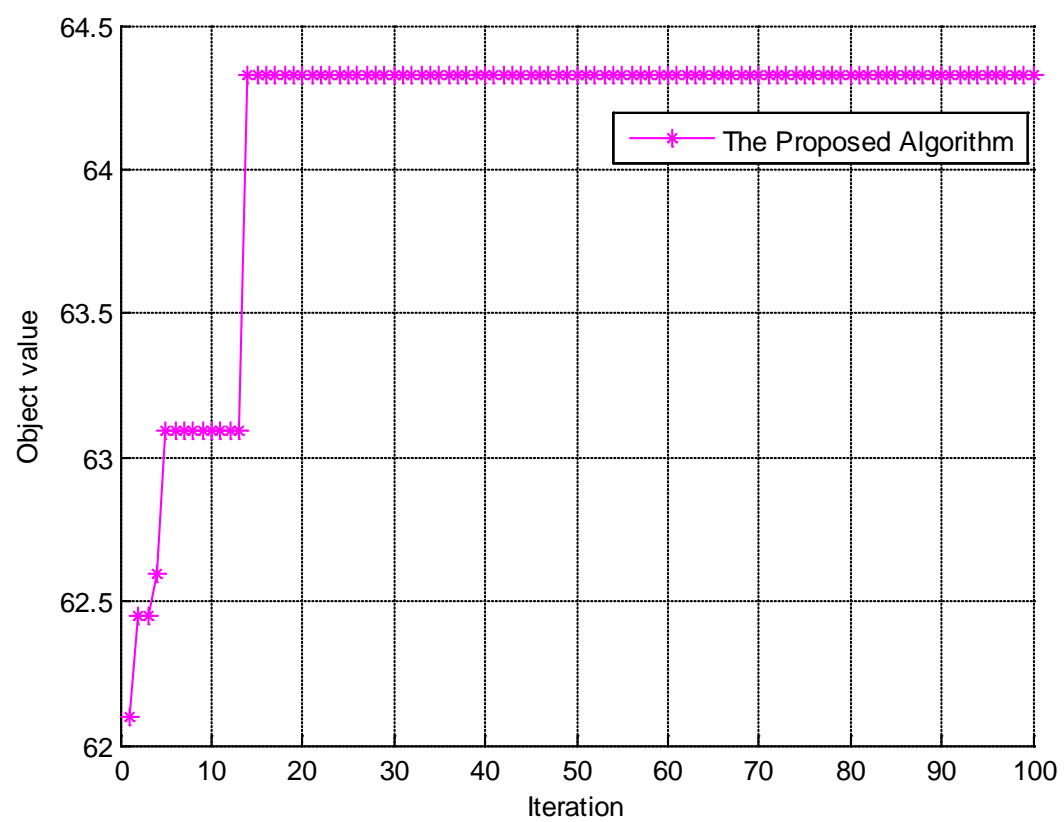

Fig. 3. The convergence of the proposed algorithm 


\section{B Energy Efficient}

In Fig. 4, a performance evaluation of the total network energy efficient versus the number of base-station is depicted. It is obvious that the proposed algorithm can achieve higher energy efficient than other two algorithms. The EEUA considers to get the energy efficiency fairness of users but it would decrease the total energy efficiency because it sacrifices large resource to improve the poor users' performance. EPAS can improve the transmission rate of edge users by improving the transmission power which would bring serious interference which will reduce the total energy efficiency.

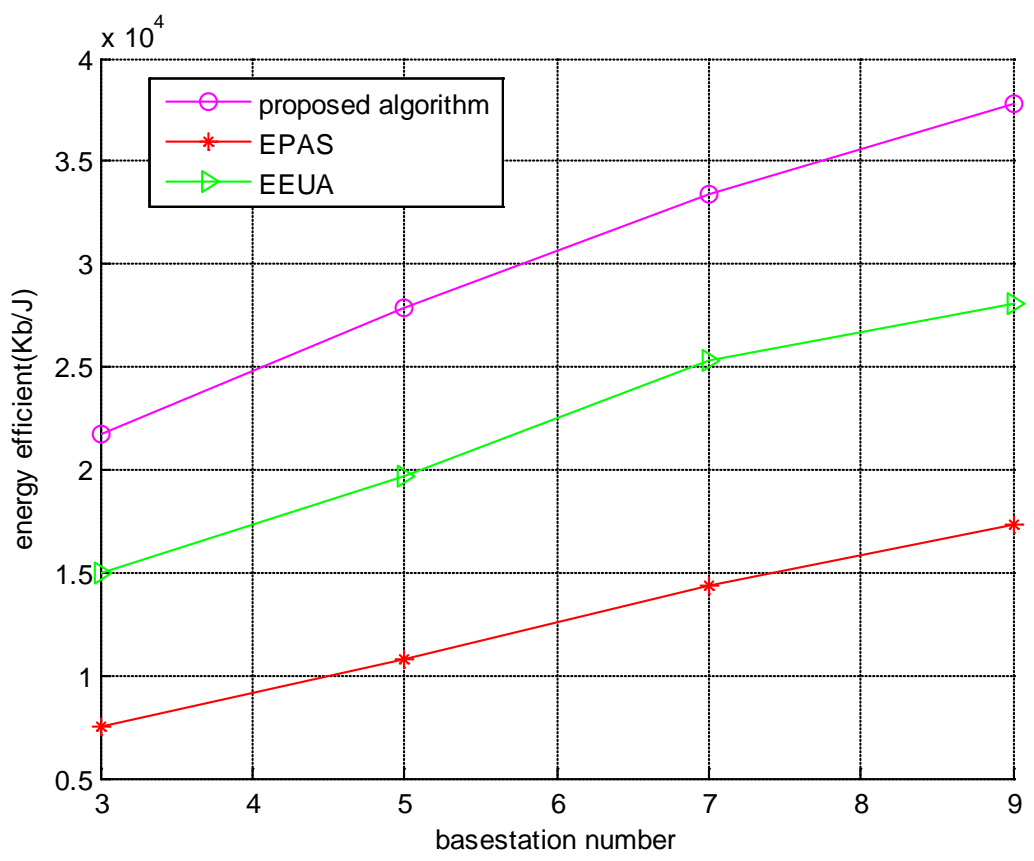

Fig. 4. Energy efficiency of system 


\section{The average MOS}

Fig. 5 demonstrates the average MOS in different algorithms with the variation of base-station number. With the increase of base-station number, users will get the higher quality of experience. However, the average MOS will increase slowly because of the serious interference. As shows in Fig. 5, we also can see that the average MOS of the proposed algorithm will be higher than that of other two algorithms since the proposed algorithm consider the users' quality of experience instead of transmission rate.

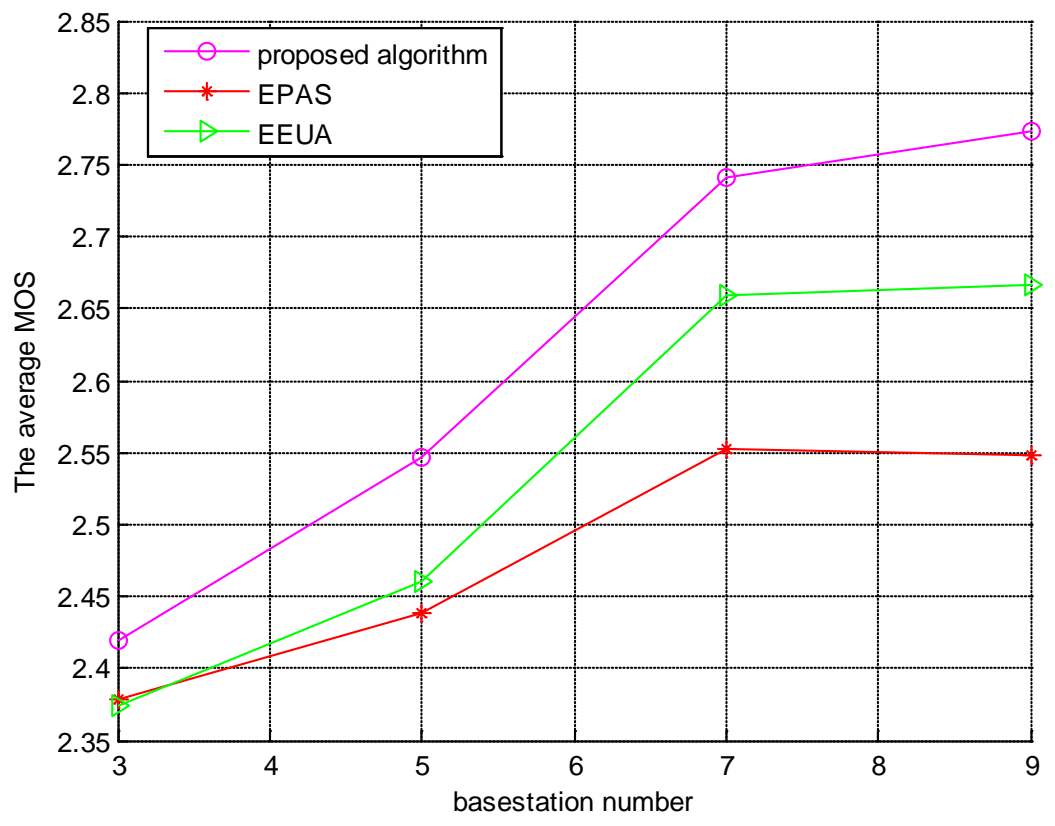

Fig. 5. The average MOS of users versus network number

Fig. 6 and Fig. 7 show the average MOS for different services in these three algorithms. It can be seen the average MOS of $C$ type is lower than that of $S$ type for its restriction of minimization transmission rate. 


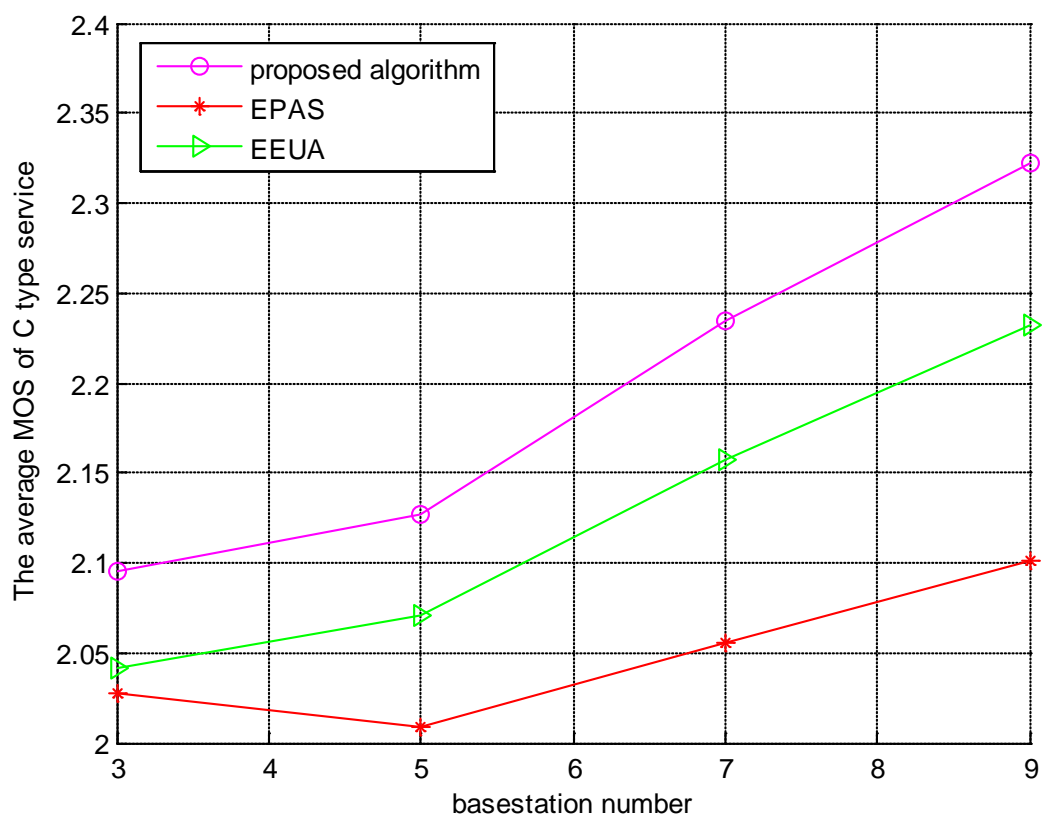

Fig. 6. The average MOS of user with C type service

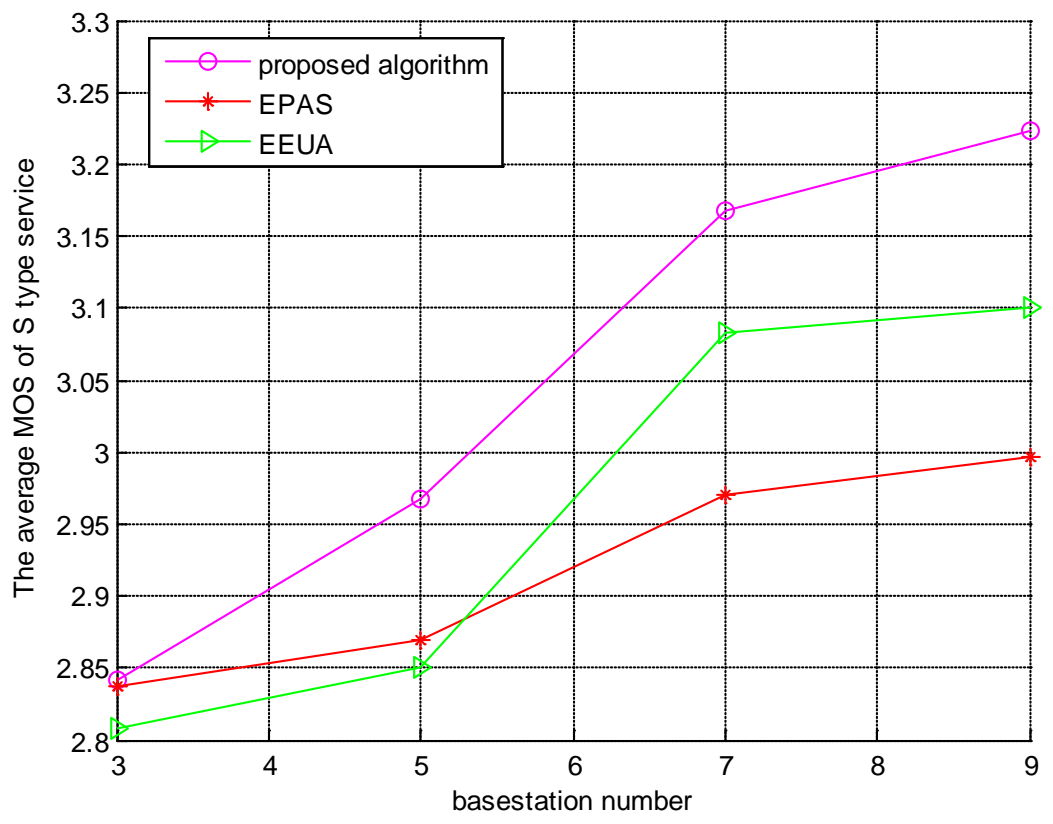

Fig. 7. The average MOS of users with S type service 
Fig. 8 shows the average MOS in different cells. From the figure, the macro-cell (base-station ID is 1) can get lower average MOS than small cells (base-station ID is 2-9). The reason is that the users in the macro-cell can receive the serious interference from small cells, while the macro-cell to small-cell user's interference is tiny. The users in small cells can get better quality of experience than users in macro-cell.

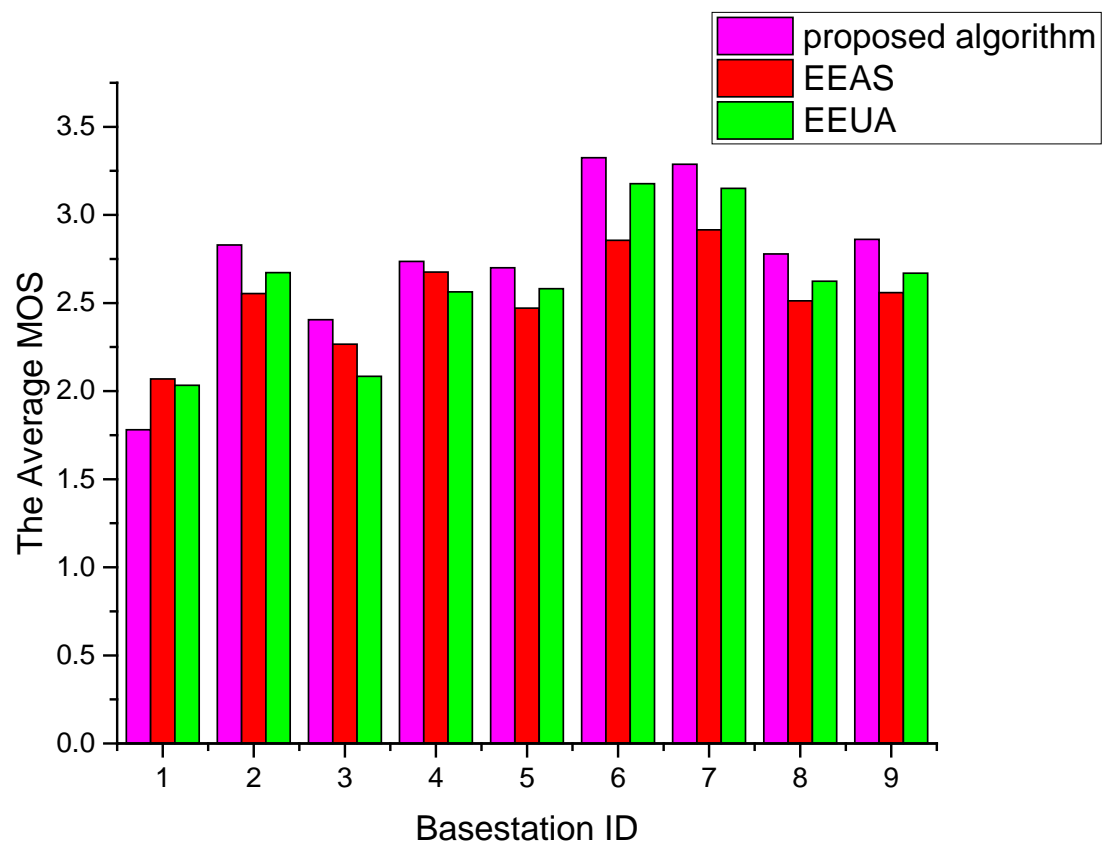

Fig. 8. The average MOS of different basestation

\section{Fairness Analysis}

In the sub-section, we use Jain's Index to measure the fairness of three algorithms. The average MOS of proposed algorithm is superior to the others but the Jain's fairness of EPAS is the highest among three algorithms in Table 3. It is because that the EPAS consider to maximize the minimization EE of users which will balance the users MOS. However, the proposed algorithm consider to promote the users MOS to near the Utopia MOS which leads to decreasing the fairness.

Table 3. Performance comparison under Jain’s Fairness factor

\begin{tabular}{lllll}
\hline & 3 & 5 & 7 & 9 \\
\hline Proposed algorithm & 0.8756 & 0.8930 & 0.8904 & 0.9084 \\
EPAS & 0.9077 & 0.9107 & 0.9141 & 0.9170 \\
EEUA & 0.8606 & 0.8836 & 0.8805 & 0.8870 \\
\hline
\end{tabular}


Fig. 9 is 10th percentile MOS (the average of the lowest 10\% MOS of users). The EPAS is outperformances in three algorithms and the proposed algorithm is second to the EEUA. The reason is the proposed algorithm does not only consider to promote the fairness but also considers to improve the average MOS, so the performance in terms of average MOS is inferior to EPAS. But as shown in Fig. 10, the EEPA sacrifices the good users' QoE to improve the poor users' QoE (The QoE in 0-1 and 4-5 is lowest in three algorithms), the proposed algorithm also can guarantee the highest good users' QoE.

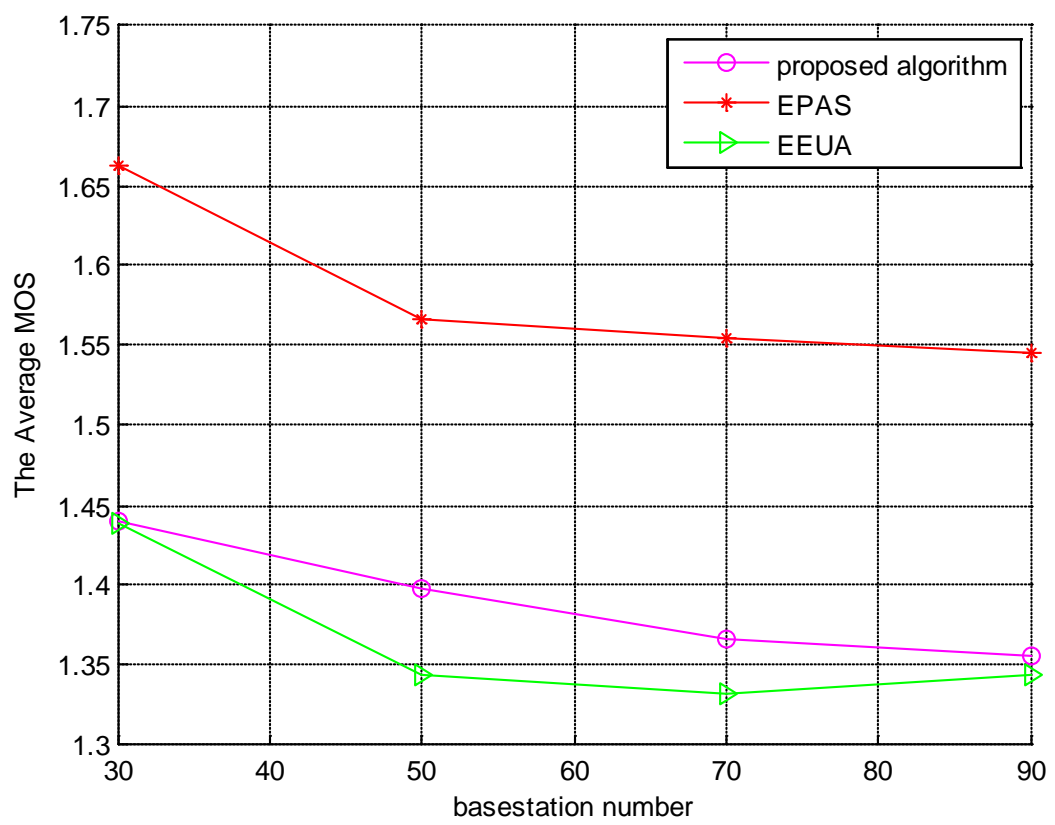

Fig. 9. $10^{\text {th }}$ percentile MOS versus network number 


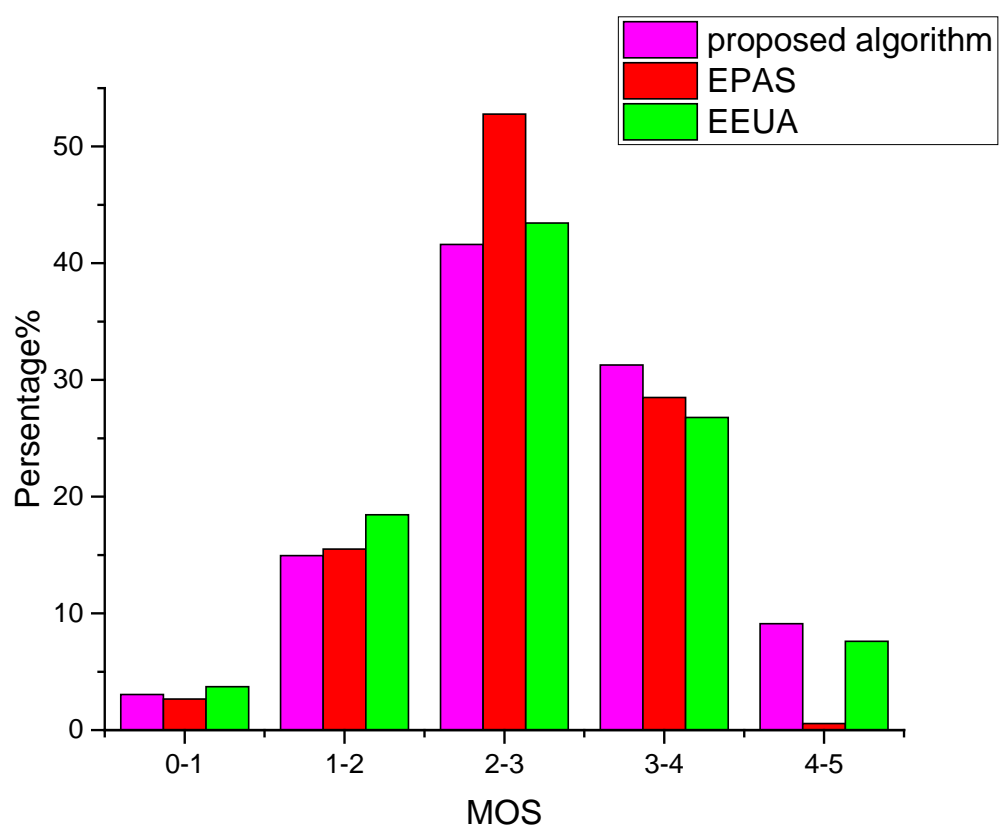

Fig. 10. The percentage of users versus the MOS

\section{Conclusions}

In this paper, we have investigated power allocation in heterogeneous networks. Our object is to improve the QEE for each user. Therefore, we formulate the optimal problem in fair manner. To find the optimal value, we use IWO to solve it. Simulation results demonstrate that the proposed algorithm can improve the QoE and EE, and achieve high QoE fairness among users. In future, we will extend our work to the scenario where there exists much relay nodes in each cell.

\section{References}

[1] N. Bhushan, J. Li, D. Malladi, R. Gilmore, D. Brenner and A. Damnjanovic, et al., "Network densification: The dominant theme for wireless evolution into 5G," IEEE Communications Magazine, vol. 52, no. 2, pp. 82-89, February 2014. Article (CrossRef Link)

[2] J. G. Andrews, S. Buzzi, W. Choi, S. V. Hanly, A. Lozano and A. C. K. Soong, et al., "What Will 5G Be?," IEEE Journal on Selected Areas in Communications, vol. 32, no. 6, pp. 1065-1082, June 2014. Article (CrossRef Link)

[3] M. Jo, T. Maksymyuk, R. L. Batista, T. F. Maciel, A. L. F. de Almeida and M. Klymash, "A survey of converging solutions for heterogeneous mobile networks," IEEE Wireless Communications, vol. 21, no. 6, pp. 54-62, December 2014. Article (CrossRef Link) 
[4] N. Ul Hasan, W. Ejaz, N. Ejaz, H. S. Kim, A. Anpalagan and M. Jo, "Network Selection and Channel Allocation for Spectrum Sharing in 5G Heterogeneous Networks," IEEE Access, vol. 4, pp. 980-992, February, 2016. Article (CrossRef Link)

[5] J. Dai and S. Wang, "QoE-driven resource allocation method for cognitive radio networks," in Proc. of 2016 IEEE International Conference on Communications (ICC), pp. 1-6, May 22-27, 2016. Article (CrossRef Link)

[6] H. Shao, W. P. Jing, X.M. Wen, Z.M. Lu, H.J. Zhang and Y.W. Chen, et al., "Joint Optimization of Quality of Experience and Power Consumption in OFDMA Multicell Networks," IEEE Communications Letters, vol. 20, no. 2, pp. 380-383, February 2016. Article (CrossRef Link)

[7] Y. S. Soh, T. Q. S. Quek, M. Kountouris and H. Shin. "Energy Efficient Heterogeneous Cellular Networks.” IEEE Journal on Selected Areas in Communications, vol. 31, no. 5, pp. 840-850, April 2013. Article (CrossRef Link)

[8] S. Bu, F. R. Yu and H. Yanikomeroglu, "Interference-Aware Energy-Efficient Resource Allocation for OFDMA-Based Heterogeneous Networks With Incomplete Channel State Information," IEEE Transactions on Vehicular Technology, vol. 64, no. 3, pp. 1036-1050, March 2015. Article (CrossRef Link)

[9] R. Cai, J. K. Zhang, T. N. Davidson, W. Zhang, K. M. Wong and P. C. Ching, "A Power Allocation Strategy for Multiple Poisson Spectrum-Sharing Networks," IEEE Transactions on Wireless Communications, vol. 14, no. 4, pp. 1785-1799, April 2015. Article (CrossRef Link)

[10] G. Ding, J. Wang, Q. Wu, Y. Yao, R. Li and H. Zhang, et al., "On the limits of predictability in real-world radio spectrum state dynamics: from entropy theory to 5G spectrum sharing," IEEE Communications Magazine, vol. 53, no. 7, pp. 178-183, July 2015. Article (CrossRef Link)

[11] S. w. Han, H. Kim, Y. Han, J. M. Cioffi and V. C. M. Leung, "A Distributed Power Allocation Scheme for Sum-Rate Maximization on Cognitive GMACs," IEEE Transactions on Communications, vol. 61, no. 1, pp. 248-256, January 2013. Article (CrossRef Link)

[12] W. Zhou, Y. Xu, T. Liang and X. Ren, "Optimal power allocation for heterogeneous network capacity maximization," China Communications, vol. 10, no. 9, pp. 97-103, September 2013. Article (CrossRef Link)

[13] Y. Gao, L. Cheng, X. Zhang, Y. Zhu, Y, Zhang. "Enhanced Power Allocation Scheme in Ultra-Dense Small Cell Network," China Communications, vol. 13, no. 2, pp. 21-9, February 2016. Article (CrossRef Link)

[14] J. Li, T. Eriksson, T. Svensson and C. Botella, "Power Allocation for Two-Cell Two-User Joint Transmission," IEEE Communications Letters, vol. 16, no. 9, pp. 1474-1477, September 2012. Article (CrossRef Link)

[15] Y. Li, Z. Zhou, M. Macuha and T. Sato., "Resource Allocation for Proportional Rate Constraint Macro Cell-edge Users in Macro-Femto Network," Transaction of the Japan Society for Simulation Technology, vol. 4, no. 4, pp. 136-144, March 2012.

Article (CrossRef Link)

[16] J. Qiu, G. Ding, Q. Wu, Z. Qian, T. A. Tsiftsis and Z. Du, et al., "Hierarchical Resource Allocation Framework for Hyper-Dense Small Cell Networks," IEEE Access, vol. 4, pp. 8657-8669, 2016. Article (CrossRef Link)

[17] J.F. Qiu, Q. H. Wu, Y. Xu and Y. Sun, "Demand-aware resource allocation for ultra-dense small cell networks: an interference-separation clustering-based solution," Transaction on Emerging Telecommunications Technologies, vol. 27, no. 8, August 2016.

Article (CrossRef Link) 
[18] D. W. K. Ng, E. S. Lo, and R. Schober, "Energy-efficient resource allocation in multi-cell OFDMA systems with limited backhaul capacity,” IEEE Transactions on Wireless Communication, vol. 11, no. 10, pp. 3618-3631, October 2012. Article (CrossRef Link)

[19] Y. Lin, S. Li, Y. Wang, C. Li, Y. Huang and L. Yang, "Energy efficient power allocation scheme in heterogeneous cellular networks," in Proc. of 2015 International Conference on Wireless Communications \& Signal Processing (WCSP), pp. 1-5, 2015. Article (CrossRef Link)

[20] A. Zappone, Z. Chong, E. A. Jorswieck and S. Buzzi, "Energy-Aware Competitive Power Control in Relay-Assisted Interference Wireless Networks," IEEE Transactions on Wireless Communications, vol. 12, no. 4, pp. 1860-1871, April 2013. Article (CrossRef Link)

[21] G. Miao, N. Himayat, G. Y. Li and S. Talwar, "Distributed Interference-Aware Energy-Efficient Power Optimization," IEEE Transactions on Wireless Communications, vol. 10, no. 4, pp. 1323-1333, April 2011. Article (CrossRef Link)

[22] Bin Han, Wenbo Wang and Mugen Peng, "A power allocation scheme for achieving high energy efficiency in two-tier femtocell networks," in Proc. of 2011 IEEE 13th International Conference on Communication Technology, pp. 352-356, 2011. Article (CrossRef Link)

[23] Y. Li, M. Sheng, X. Wang, Y. Zhang and J. Wen, "Max-Min Energy-Efficient Power Allocation in Interference-Limited Wireless Networks," IEEE Transactions on Vehicular Technology, vol. 64, no. 9, pp. 4321-4326, September 2015. Article (CrossRef Link)

[24] D. Yuan, M. Song, Y. Teng, D. Ma, X. Wang and G. Lu, "QoE-oriented resource allocation for multiuser-multiservice femtocell networks," China Communications, vol. 12, no. 10, pp. 27-41, Oct. 2015. Article (CrossRef Link)

[25] Chen, Feng, X. Qin, and G. Wei. "QoE Optimized Resource Allocation in Multiuser OFDM Systems," Przeglad Elektrotechniczny, vol. 88, no. 7, pp.328-331, 2012. Article (CrossRef Link)

[26] Y. H. Cho, H. Kim, S. H. Lee and H. S. Lee, "A QoE-Aware Proportional Fair Resource Allocation for Multi-Cell OFDMA Networks," IEEE Communications Letters, vol. 19, no. 1, pp. 82-85, January 2015. Article (CrossRef Link)

[27] S. Thakolsri, S. Khan, E. Steinbach and W. Kellerer. "QoE-driven cross-layer optimization for high speed downlink packet access,” Journal of Communications, vol. 4, no. 9, pp. 669-680, October 2009. Article (CrossRef Link)

[28] Q. T. Nguyen-Vuong, Y. Ghamri-Doudane and N. Agoulmine, "On utility models for access network selection in wireless heterogeneous networks," in Proc. of NOMS 2008 - 2008 IEEE Network Operations and Management Symposium, pp. 144-151, 2008. Article (CrossRef Link)

[29] W. Dinkelbach, “On nonlinear fractional programming,” Managemet Science, vol. 13, no. 7, pp. 492-498, March 1967. Article (CrossRef Link)

[30] Haijun Zhang, Chunxiao Jiang, Xiaoli Chu, Xiangming Wen, Meixia Tao. "Resource Allocation in Spectrum-Sharing OFDMA Femtocells With Heterogeneous Service," IEEE Transactions on Communications, vol. 62, No. 7, pp. 2366-2377. July 2014. Article (CrossRef Link)

[31] G. Yu, Y. Jiang, L. Xu and G. Y. Li, "Multi-Objective Energy-Efficient Resource Allocation for Multi-RAT Heterogeneous Networks," IEEE Journal on Selected Areas in Communications, vol. 33, no. 10, pp. 2118-2127, Oct. 2015. Article (CrossRef Link)

[32] H. Chen, Y. Zhou, S. He, X. Ouyang, and P. G. Guo. "Invasive Weed Optimization Algorithm for Solving Permutation Flow-Shop Scheduling Problem,” Journal of Computational and Theoretical Nanoscience, vol. 10, No. 3, pp. 708-713, March 2013. Article (CrossRef Link) 

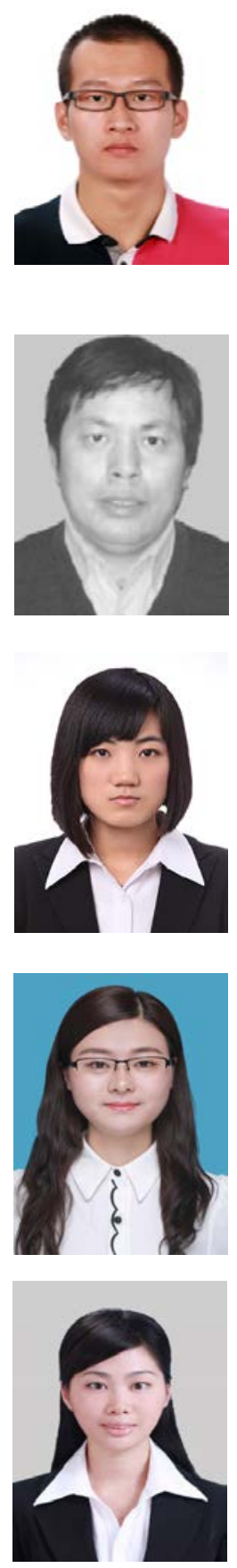

Shiyu Ji received the B.S. degree in 2013 and now is a candidate for Ph.D. degree in Electrical Information Technology of North China Electric Power University, Beijing, China. His research interests include wireless networks and communication technology in power system.

Liangrui Tang received the Ph.D. degree in Communication and Information System from Beijing University of Posts and Telecommunications. Now, he is a Professor in State Key Laboratory of Alternate Electrical Power System with Renewable Energy Sources of North China Electric Power University, focusing on the research of communication in power system, wireless communications and optical network communication.

Yanhua He received the B.S. degree in 2015 and now is a candidate for Ph.D. degree in Electrical Information Technology of North China Electric Power University, Beijing, China. Her research interests include resource allocation in heterogeneous network.

Shuxian Li received the B.S. degree in 2016 and now is a candidate for master degree in Electrical Information Technology of North China Electric Power University, Beijing, China. Her research interests include wireless networks resource management.

Shimo Du received the M.S. degree in Electronic and Information Engineering from North China Electric Power University, Beijing, China, in 2014. And now, she is currently an engineer in China mobile communication corporation Hangzhou branch. Her research interests include congestion control, network traffic analysis and communication technology in power system. 